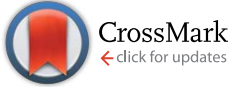

Cite this: RSC Adv., 2017, 7, 16836

Received 12th January 2017

Accepted 3rd March 2017

DOI: $10.1039 / c 7 r a 00509 a$

rsc.li/rsc-advances

\section{Hollow Au loaded with kanamycin for pharmacological and laser-triggered photothermal sterilization $\dagger$}

\author{
Xing Zhang, ${ }^{\text {a }}$ Yuxia Li, ${ }^{\text {b }}$ Juhui Qiu, ${ }^{c}$ Dandan Zhou, ${ }^{\text {b }}$ Minghao Zhang, ${ }^{\text {d }}$ Lan Tang, ${ }^{\text {b }}$ \\ Guoming Xie ${ }^{\star a}$ and Hua Xiang ${ }^{\star a}$
}

\begin{abstract}
Effective and appropriate use of existing antibiotics is imperative because of the problem of antibiotic resistance. Driven by this need, a platform of hollow Au loaded with kanamycin (hAu-kana) was produced. This platform has the ability to target drug delivery and facilitate photothermal sterilization. Hollow Au was synthesized by galvanic replacement reaction. We then covalently attached anti-E. coli to hollow Au modified with the ligands SH-PEG and L-cysteine. SH-PEG prevents the aggregation of AuNPs, and the carboxyl group of the L-cysteine further links with IgG. The dual-ligand hollow Au can be targeted to deliver the drug after modification with the anti-E. coli, thus improving the sterilization efficiency. This was followed by loading the model drug kanamycin (hAuNPs-anti-E. coli-kana) to facilitate photothermal treatment. The bactericidal effect on the bacterial strain was investigated by incubating E. coli with the hybrid and eventually subjecting it to laser irradiation $\left(650 \mathrm{~nm}, 200 \mathrm{~mW} \mathrm{~cm}{ }^{-2}\right.$, diameter $2 \mathrm{~mm}, 40 \mathrm{~min}$ ). Compared with E. coli treated with hAuNPs-anti-E. coli-kana alone, irradiation of $E$. coli treated with hAuNPs-anti-E. coli-kana with laser has obvious synergistic effect and made the sterilization more effective. Taken together, this hybrid suggests a new method for achieving synergistic bactericidal effect.
\end{abstract}

\section{Introduction}

The problem of antibiotic resistance is not only man-made; resistance genes have been present in the bacterial population long before humans started to use antibiotics to fight bacterial infections..$^{1,2}$ Despite an armamentarium of antibacterial, antifungal and antiviral agents for the treatment of most infectious diseases, the biggest threats today are resistant pathogens, especially those in hospitals. ${ }^{3-6}$ In order to meet the growing problem of antibiotic resistance among pathogens, the development of new treatments for infectious diseases that will ensure effective and appropriate use of existing antibiotics is imperative.

Hollow Au nanoparticles (hAuNPs) have the unique characteristics of small size (outer diameter, $\sim 50 \mathrm{~nm}$ ), spherical

${ }^{a}$ Key Laboratory of Laboratory Medical Diagnostics, Chinese Ministry of Education, Department of Laboratory Medicine, Chongqing Medical University, Chongqing, 400016, China. E-mail: xianghuacq@163.com

${ }^{b}$ The First Affiliated Hospital of Chongqing Medical University, Chongqing 400016, China

'State Key Laboratory of Membrane Biology, Tsinghua-Peking Center for Life Sciences, School of Life Sciences, Tsinghua University, Beijing 100084, China

${ }^{d}$ Center for Lab Teaching \& Management, Chongqing Medical University, Chongqing 400016, China

$\dagger$ Electronic supplementary information (ESI) available. See DOI: $10.1039 / \mathrm{c} 7 \mathrm{ra00509a}$ shape, a hollow interior and, especially, good biocompatibility, and they present promising application prospects in various fields. ${ }^{7-11}$ As a substitute for complex processing with special equipment requirements, a more simple and effective method can allow chloroauric acid $\left(\mathrm{HAuCl}_{4}\right)$ to undergo spontaneous transformation in sodium borohydride $\left(\mathrm{NaBH}_{4}\right)$ solution and produce hollow structures. ${ }^{12,13}$ The obtained hAuNPs are most suitable for serving as drug carriers with hollow structure. ${ }^{14}$ Meanwhile, the characteristics of the hAuNP surface present the possibility of covalent modification with other biomolecules. Taking into account the biocompatibility, load and target problems of nanomaterials in application, in this work, we chose the hAuNPs and antibody modification of hAuNPs. At the same time, under irradiation conditions, the hAuNPs containing chemical drugs can provide the usual chemical and physical sterilization functions.

It is very important to improve the stability of hAuNPs in this system. $O$-[2-(3-Mercaptopropionylamino)ethyl]-O'-methylpolyethylene glycol (SH-PEG) is a commonly used ligand to stabilize metal nanoparticles (NPs) in different $\mathrm{pH}$ and salt solutions. ${ }^{15-18}$ However, due to the SH-PEG surface not having functional groups, it cannot connect with antibodies, enzymes or others, limiting its application. Furthermore, carboxyl functionalization seems to attenuate toxicity. ${ }^{19}$ Because of this, we used the method of dual-ligand modification of hAuNPs, in which the surface gains carboxyl groups to react with the antibody, and 
SH-PEG coating, which greatly improved its stability in the medium. ${ }^{20,21}$ HAuNPs with a mixed monolayer of SH-PEG and Lcysteine (1:10 volume ratio) covalently conjugated to IgG through $\mathrm{Au}-\mathrm{NH}_{2}$. HAuNPs modified with the antibody had targeted drug transport property, which could selectively reduce the drug's side effects.

Nanomaterial-mediated photothermal therapy (PTT) is a promising strategy for sterilization. Its principle is mainly via damage to the integrity of the cell structure by degrading the nuclear membrane and endoplasmic reticulum. ${ }^{22-26}$ This type of light-triggered treatment modality has improved selectivity and lowered side effects. Various kinds of NPs have been reported to be able to enter cells by endocytic pathways. ${ }^{27-29}$ AuNPs are known to be nontoxic and nonimmunogenic. AuNPs also have the characteristics of light absorption. ${ }^{30}$ Here, we used the photothermal effect of hAuNPs to reach a synergistic bactericidal effect with the drug.

In this study, we report a hybrid bactericidal material, hAuNPs that are dual-ligand modified, anti-E. coli conjugated and kanamycin-loaded (hAuNPs-anti-E. coli-kana), which could selectively target bacteria and kill them by combining the effects of kanamycin and photothermal therapy. Meanwhile, the hybrid could reduce the risk of abuse of broad-spectrum antibiotics. In addition, the synergistic effect of the chemical drug and physical sterilization of hAuNPs-anti-E. coli-kana is an improvement on the bactericidal effect.

\section{Experimental section}

\section{Instrumentation}

UV-vis absorption spectra are obtained with a spectrophotometer (Thermo Scientific, Nanodrop 1000, USA). Magnetic stirrer is used in material synthesis (Shanghai Sile instrument Co. Ltd., S21-3, China). The concentration of bacteria in the suspension is measured using a cell density meter (Amersham Biosciences, Ultrospec 10, USA). Zetasizer is used to measure the size and the zeta potential of the NPs (Malvern, Nano ZS-90, UK).

Other apparatus used also includes the following: air clean bench (Aetna Corp, SW-CJ-1FD, China), the classical multiamplitude orbital shaker (Zhicheng Analytical Instrument Manufacturing Co., Ltd., ZWY-100H, China), digital thermometer (Wanda, TP 101, China), low-temperature incubator (Shellab, SRI3-2, USA), Labo autoclave (Sanyo, MLS-3780, Japan), confocal fluorescence microscope (Nikon, Eclipse 80i, Japan), laser head (Diamond Electronics Co., Ltd., $650 \mathrm{~nm}, 200 \mathrm{~mW}$ $\mathrm{cm}^{-2}$, China), pH meter (Leici, PHS-3C, China), scanning electron microscopy (SEM) (Hitachi, S-3000 N, Japan), transmission electron microscopy (TEM) (Hitachi, 7500, Japan) for characterization of NPs, and TEM (JEOL, JEM-1400Plus, Japan) for the study of bacterial slices.

\section{Reagents}

Cobalt chloride, sodium borohydride, $\mathrm{O}$-[2-(3-mercaptopropionylamino)ethyl]- $O^{\prime}$-methylpolyethylene glycol (SH-PEG), and $N$ hydroxysuccinimide (NHS) were purchased from Sigma-Aldrich. Sodium citrate, chloroauric acid, kanamycin, culture dish, crystal violet staining solution, Gram's iodine, and Safranin O were purchased from Sangon Biotech. L-Cysteine was purchased from Bio Basic, Inc. 1-Ethyl-3-(3-dimethylaminopropyl) carbodiimide (EDC) was purchased from Damas-Beta. Escherichia coli was purchased from American Type Culture Collection (ATCC) (Fig. S1†). Antibody to E. coli (anti-E. coli) was purchased from Abcam.

The Luria-Bertani (LB) nutrient solution was prepared by dissolving sodium chloride, tryptone and yeast extract in deionized (DI) water. LB solid medium was obtained by adding agar. Phosphate-buffered saline (PBS) was prepared by mixing the solutions of $\mathrm{NaH}_{2} \mathrm{PO}_{4}$ and $\mathrm{Na}_{2} \mathrm{HPO}_{4}$. DI water purified through a Millipore system ( $\geq 18 \mathrm{M} \Omega \mathrm{cm}$ ) was employed in all runs.

\section{Preparation of the hAuNPs}

HAuNPs were synthesized according to a previously reported method with a little modification..$^{31}$ Briefly, cobalt nanoparticles (CoNPs) were first synthesized by deoxygenating $100 \mathrm{~mL}$ DI water containing $400 \mu \mathrm{L}$ of $0.1 \mathrm{M}$ sodium citrate and $100 \mu \mathrm{L}$ of $0.4 \mathrm{M}$ cobalt chloride, and bubbling the solution with nitrogen $(\sim 20-30$ min). Sodium borohydride ( $100 \mu \mathrm{L}, 1 \mathrm{M})$ was then added. The slightly pink solution turned light grey and then brown on the addition of sodium borohydride, indicating the reduction of Co(II) and the formation of CoNPs. The solution was allowed to react for $45 \mathrm{~min}$ under constant nitrogen flow before the addition of gold. Thereafter, $30 \mathrm{~mL}$ of the CoNP solution was transferred immediately to a vortexing solution of $10 \mathrm{~mL}$ DI containing 15 to $35 \mu \mathrm{L}$ of $0.1 \mathrm{M}$ chloroauric acid. The cobalt immediately reduced the gold ions onto the surface of CoNPs, while at the same time, it was oxidized to cobalt oxide. Any remaining cobalt core was further oxidized by air, resulting in the final product, hAuNPs.

\section{Preparation of hAuNPs-anti-E. coli}

SH-PEG $(10 \mu \mathrm{L})$ and L-cysteine $(100 \mu \mathrm{L})$ were added to the hAuNP solution, stirred for $30 \mathrm{~s}$, and then stored at $4{ }^{\circ} \mathrm{C}$ for further use.

After the activation of carboxylic acid group with $2 \mathrm{mM}$ of EDC and $5 \mathrm{mM}$ of NHS in $0.1 \mathrm{M}$ of PBS, $10 \mu \mathrm{L}$ of the antibody was added to the pre-modified hAuNP dispersion. The mixture was shaken for $2 \mathrm{~h}$ at room temperature. After centrifugation (5000 rpm s${ }^{-1}, 15 \mathrm{~min}, 4^{\circ} \mathrm{C}$ ) to remove excess antibodies, the precipitate was resuspended in PBS solution and stored at $4{ }^{\circ} \mathrm{C}$, to be used within a week.

\section{Loading of kanamycin on hAuNPs-anti-E. coli}

Kanamycin was loaded into hAuNPs-anti-E. coli $\left(1 \mathrm{mg} \mathrm{mL}{ }^{-1}\right)$ by mixing the NPs with a kanamycin solution $\left(1 \mathrm{mg} \mathrm{mL}^{-1}\right)$ in PBS (0.1 M, $1 \mathrm{~mL})$. The mixture was gently shaken at room temperature for $24 \mathrm{~h}$ in the dark. Free kanamycin was removed by washing with PBS followed by centrifugation, done several times.

\section{Characterization of hAuNPs}

UV-vis absorption spectra were used for analysis of sample composition. The zeta potentials of the samples were determined on a Zetasizer at $25{ }^{\circ} \mathrm{C}$. The structure and morphology of samples were observed with TEM. 


\section{Verification of photothermal properties of hAuNPs}

To verify the photothermal effect of hAuNPs, $10 \mathrm{~mL}$ of $\mathrm{SH}$ PEGylated hAuNPs $(0.5 \mathrm{mM})$ in a glass vial were irradiated by $650 \mathrm{~nm}$ laser with an intensity of $200 \mathrm{~mW} \mathrm{~cm}^{-2}$ (diameter 2 $\mathrm{mm}$ ) for $40 \mathrm{~min}$, and the temperature changes were measured by a digital thermometer.

\section{Cytotoxicity assay}

E. coli were inoculated in LB broth and grown at $37^{\circ} \mathrm{C}$ for $16 \mathrm{~h}$, then stored at $4{ }^{\circ} \mathrm{C}$ for use. E. coli (0.5 MCF) were diluted with LB broth to $1 \times 10^{5} \mathrm{cfu} \mathrm{mL}^{-1}$ and stored as the original bacterial solution. The as-prepared $E$. coli solution $(50 \mu \mathrm{L})$ was mixed with diverse concentrations of hAuNPs $(0.3 \mathrm{mM}, 0.5 \mathrm{mM}, 1.0 \mathrm{mM}$, $2.0 \mathrm{mM}, 30 \mu \mathrm{L}$, respectively) and diluted with $\mathrm{LB}$ broth to $500 \mu \mathrm{L}$. Then, $100 \mu \mathrm{L}$ of the bacterial solutions were placed on LB solid medium and cultured at $37^{\circ} \mathrm{C}$ for $16 \mathrm{~h}$. The bacterial toxicity was evaluated by counting the number of bacterial colonies.

\section{Photothermal treatment of bacteria by the hAuNPs-anti-E. coli-kana nanocapsules}

To evaluate the bactericidal property of the hAuNPs-anti-E. colikana nanocapsules, E. coli (0.5 MCF) solution was diluted with broth to $1 \times 10^{5} \mathrm{cfu} \mathrm{mL}^{-1}$ and stored as original bacterial solution. The as-prepared $E$. coli solution $(50 \mu \mathrm{L})$ was mixed with hAuNPs-anti-E. coli-kana nanocapsules $(30 \mu \mathrm{L})$ and diluted with LB broth to $500 \mu \mathrm{L}$. Three samples of the same solution were prepared in parallel. The obtained bacterial mixture was irradiated for $40 \mathrm{~min}$ under laser, then shaken for $10 \mathrm{~min}$. Bacteria from the above solution $(100 \mu \mathrm{L})$ was coated on the LB solid medium, and the number of bacterial colonies was counted after $37{ }^{\circ} \mathrm{C}$ culture for $16 \mathrm{~h}$. Control experiments were performed in parallel, i.e., the bacteria diluted with LB broth in the absence of hAuNPs-anti-E. coli-kana nanocapsules. To illustrate that the combination of chemical drug and physical photothermal bactericidal effect caused bacterial death, the presence of kanamycin-only and bare hAuNPs under laser irradiation were investigated in accordance with the steps described in the previous procedures.

\section{TEM images of hAuNPs-anti-E. coli-kana-treated bacteria}

The prepared hAuNPs-anti-E. coli-kana was added into the bacterial solution in a flask and incubated for $4 \mathrm{~h}$ at $37{ }^{\circ} \mathrm{C}$ to induce receptor-mediated combination of NPs. Untreated bacterial solution was prepared as a control. Then, TEM samples were prepared. The bacteria were fixed in a fixing solution overnight at $4{ }^{\circ} \mathrm{C}$. The fixed bacteria were dehydrated in serially diluted ethanol (30, 50, 70, 80, 90 and 100\%). After dehydration, the bacteria were infiltrated at $70{ }^{\circ} \mathrm{C}$ overnight. The samples were cut into ultrathin sections and mounted on a copper grid. Then, the sections were stained and observed with a JEM-1400Plus TEM.

\section{Results and discussion}

\section{Fabrication of the hAuNPs-anti-E. coli-kana}

The overall schematic diagram for the synthesis of targetspecific hAuNPs-anti-E. coli-kana is shown in Scheme 1. First, hAuNPs $(\sim 50 \mathrm{~nm}$ in diameter) were prepared by galvanic replacement reaction. During the galvanic replacement reaction, the CoNPs were galvanically replaced by gold ions, accompanying the generation of a hollow interior, resulting in an increase of drug loading capacity. As-prepared hAuNPs were coated with SH-PEG/L-cysteine (molar ratio $1: 10$ ) to provide biocompatibility and bio-conjugation sites. After modification, anti-E. col $i$ was conjugated to the carboxyl group of pre-modified hAuNPs by EDC/NHS coupling method. Lastly, kanamycin was introduced into the hAuNPs-anti-E. coli carrier. Kanamycin was loaded by external (DO, "drug outside" on the exterior surface area of hAuNPs) and internal loading (DI, "drug inside" the cavity of hAuNPs) methods to the hAuNPs.

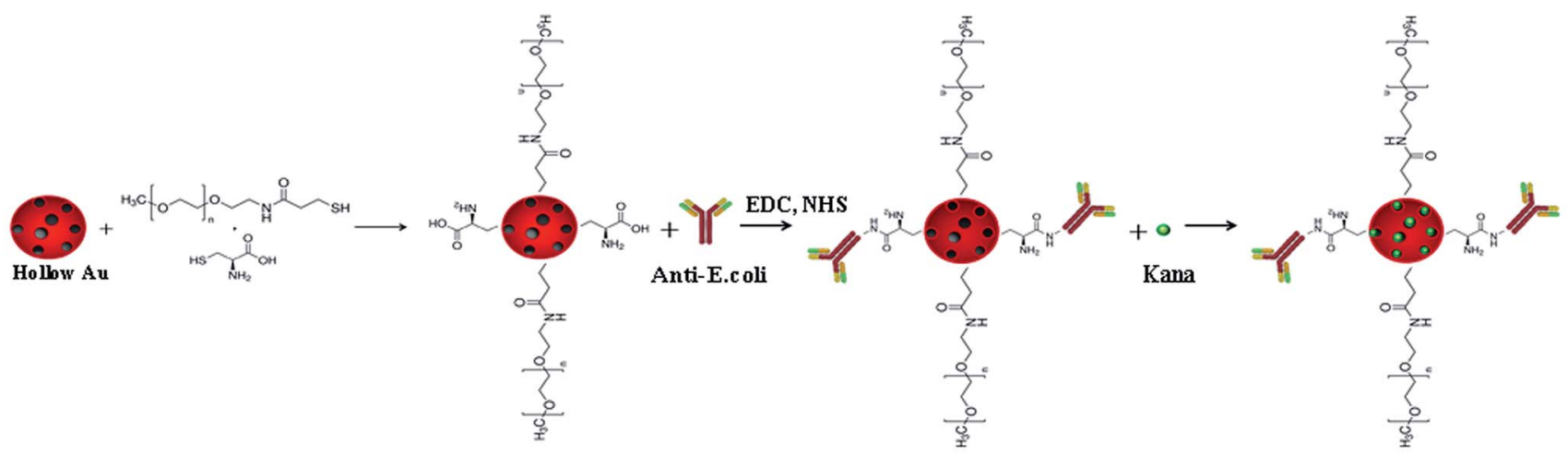

a) $\mathrm{HS}_{\mathrm{NH}_{2}}^{\mathrm{O}} \mathrm{OH}, \mathrm{L}(+)-$ Cysteine

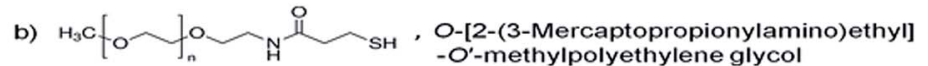

Scheme 1 Schematic illustration for the preparation of hAuNPs-anti-E. coli-kana. After the synthesis of hAuNPs by galvanic replacement reaction, hAuNPs were modified with SH-PEG and L-cysteine to allow conjugation of the $E$. coli antibody and increase biocompatibility. Next, $E$. coli antibody was conjugated through activation of the carboxyl groups at the surface of pre-modified hAuNPs, followed by kanamycin loading. 


\section{Characterization of the hAuNPs-anti-E. coli-kana}

Homogeneous distribution of the prepared hAuNPs dispersed in DI water was observed in the TEM image (Fig. 1A). Fig. 1B is an amplification of the hAuNP image, and obvious hollow structure is seen. The hAuNPs were uniformly dispersed in the solution without obvious aggregation, mainly due to the negative charge on the surface of the particles. Particle diameter analysis was measured at $25^{\circ} \mathrm{C}$ with the Zetasizer Nano ZS90, as shown in Fig. 1C. Zetasizer Nano ZS90 uses dynamic light scattering (DLS) to measure particle size with a 90 degree scattering angle. The prepared hAuNPs had relatively good stability, as shown in Fig. S2. $\dagger$

The plasmonic spectra of the hAuNPs were measured by UVvis spectrometry, as shown in Fig. 2A. The plasmonic band of the hAuNPs formed a peak at $630 \mathrm{~nm}$, which was attributed to the hAuNPs, in comparison with that of the CoNPs, due to galvanic replacement. Strong absorbance of hAuNPs could be related to a high potential for heat generation upon irradiation with laser. ${ }^{32,33}$ The antibody covalent-bonding and kanamycinloading properties of hAuNPs-anti-E. coli-kana were evaluated. UV-vis spectra revealed a newly appeared peak at around $280 \mathrm{~nm}$, corresponding to anti-E. coli, after antibody bonding. After kanamycin loading, the curve changed to (c) (Fig. 2B). The hollow structure of hAuNPs promoted kanamycin loading inside its interior spaces.
To further verify the successful synthesis, the surface charges of these NPs at different stages were determined by zeta potential measurement. ${ }^{34-36}$ The zeta potential of the samples after incubation are provided in Fig. 3. A significant transition of the zeta potential from negative $-25.5 \mathrm{mV}$ to $-10.8 \mathrm{mV}$ was observed after each step of modification, confirming the successful preparation of hAuNPs-anti-E. coli-kana.

\section{Condition optimization}

In order to prove that the hAuNPs-anti-E. coli-kana was suitable for the bactericidal effect, we evaluated the bactericidal properties of hAuNPs and kanamycin separately. Fig. 4A shows that at a hAuNP concentration of $2.0 \mathrm{mM}$, about $80 \%$ of $E$. coli was still viable. On the other hand, the increasing kanamycin concentration from $0-10 \mathrm{mg} \mathrm{mL}^{-1}$ led to improvement of the bactericidal effect, and the survival rate of $E$. coli decreased to $6 \%$ at $5 \mathrm{mg}$ $\mathrm{mL}^{-1}$ (Fig. 4B). For the purpose of studying the bactericidal ability of the hAuNPs-anti-E. coli-kana hybrid, it is necessary to maintain the survival of $E$. coli. In this respect, the concentration of hAuNPs and kanamycin was set to $0.5 \mathrm{mM}$ and $1 \mathrm{mg} \mathrm{mL} \mathrm{m}^{-1}$, respectively.

\section{Photothermal property of hAuNPs}

HAuNPs-anti-E. coli-kana has three functions: targeting specific bacteria via the anti-E. coli, photothermal treatment by laser irradiation, and drug delivery using the hAuNPs (Scheme 2).
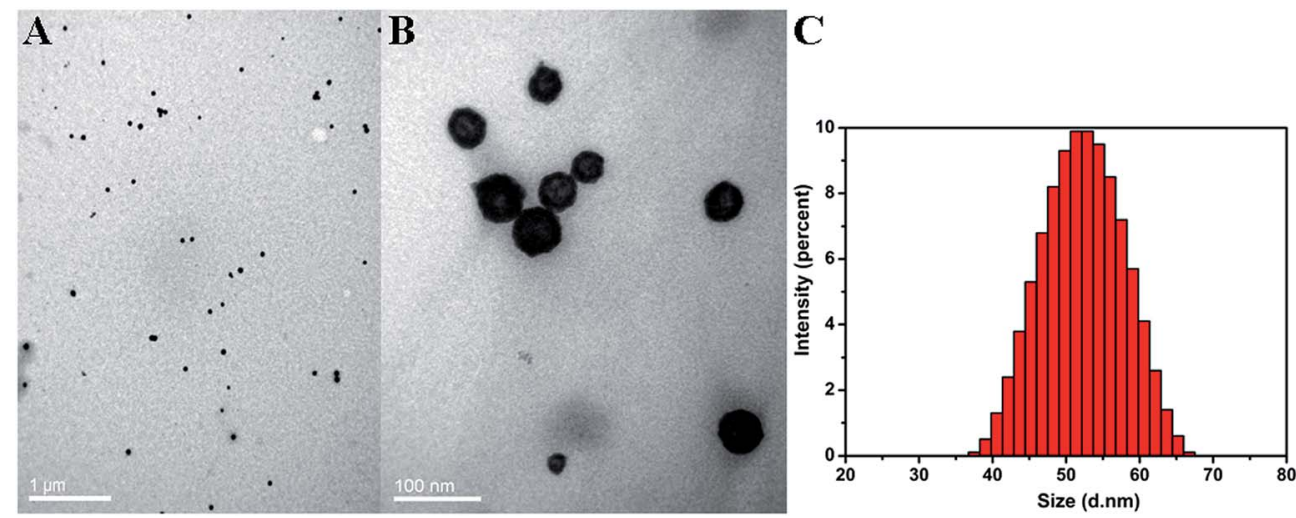

Fig. 1 (A) TEM and (B) amplification images, and (C) particle diameter analysis of hAuNPs at $25^{\circ} \mathrm{C}$.
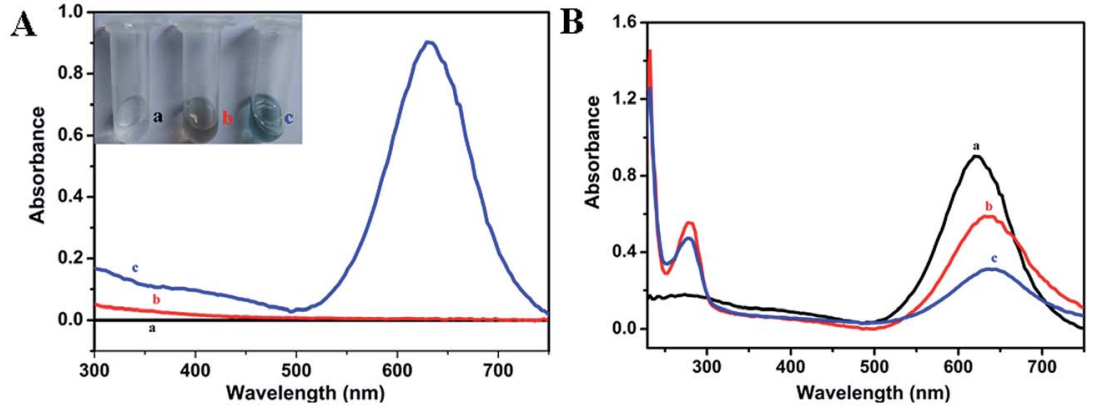

Fig. 2 (A) UV-vis spectra of (a) DI water, (b) CoNPs and (c) hAuNPs. Illustration is the solution color of the corresponding curve. (B) UV-vis spectra of (a) hAuNPs and (b) hAuNPs conjugated with anti-E. coli through dual-ligand and (c) hAuNPs-anti-E. coli loaded with kanamycin. 


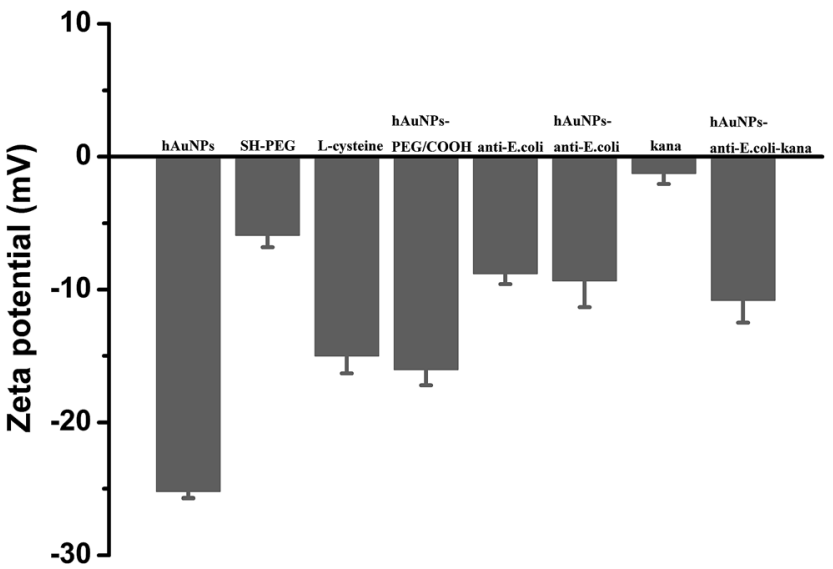

Fig. 3 The corresponding zeta potential measured at each step of the preparation process. The error bars represent the standard deviation of three measurements.

Receptor-mediated delivery for target cells is more effective than non-specific. ${ }^{37-40}$ After targeting, the bacteria were exposed to the laser. Meanwhile, the targeted bacteria were subjected to photothermal effects and the drug.

To confirm the photothermal effect, temperature changes of the hAuNP dispersion in DI water were measured upon irradiation using a laser with a wavelength of $650 \mathrm{~nm}$. As shown in Fig. 5, the temperature of the hAuNP dispersion $(0.5 \mathrm{mM})$ gradually increased from $29.1{ }^{\circ} \mathrm{C}$ to $43.0^{\circ} \mathrm{C}\left(\Delta T=13.9^{\circ} \mathrm{C}\right)$ upon

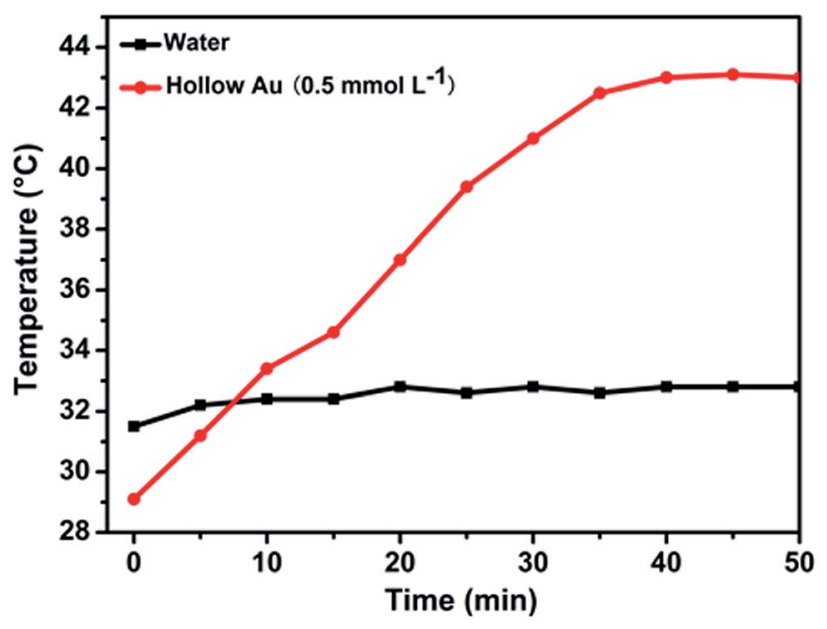

Fig. 5 Temperature change of hAuNP suspension $\left(0.5 \mathrm{mmol} \mathrm{L^{-1 }}\right)$ under laser irradiation $\left(650 \mathrm{~nm}, 200 \mathrm{~mW} \mathrm{~cm}^{-2}\right)$ for $40 \mathrm{~min}$. DI water was used as control under the same conditions.

irradiation ( $650 \mathrm{~mW} \mathrm{~cm} \mathrm{~cm}^{-2}, 40 \mathrm{~min}$ ), whereas the temperature of DI water rose by less than $2{ }^{\circ} \mathrm{C}$ after irradiation. This elevation in temperature could induce bacterial death and thus the combined effects on bacteria of radiation and drug sensitivity.

\section{Bactericidal properties of hAuNPs-anti-E. coli-kana in vitro}

The bactericidal effect of hAuNPs-anti-E. coli-kana incubated with $E$. coli, after laser irradiation, was measured by the number
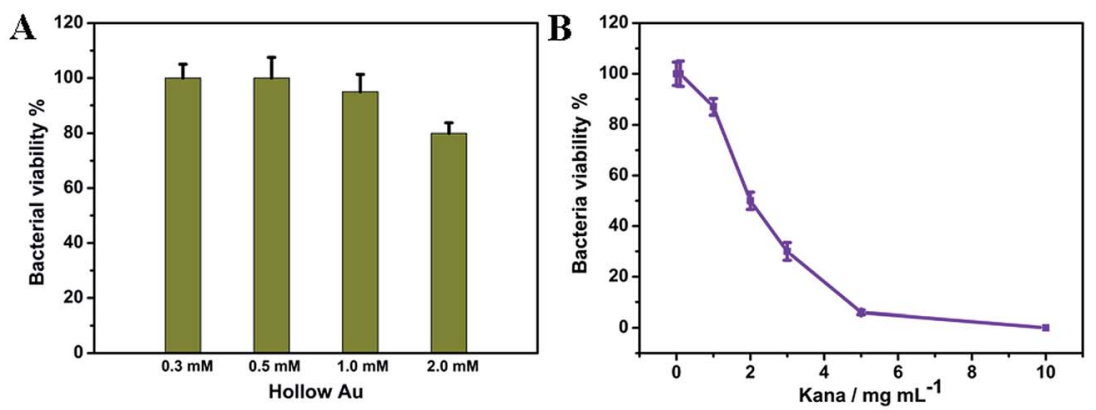

Fig. 4 The viability of $E$. coli with the concentration of (A) hAuNPs and (B) kanamycin. The concentration of $E$. coli: $1 \times 10^{5} \mathrm{cfu} \mathrm{mL}^{-1}$; hAuNPs: $0.3 \mathrm{mM}, 0.5 \mathrm{mM}, 1.0 \mathrm{mM}, 2.0 \mathrm{mM}$; kanamycin: 0, 0.1, 1.0, 2.0, 3.0, 5.0, $10.0 \mathrm{mg} \mathrm{mL}^{-1}$. The data were averaged from three parallel measurements.

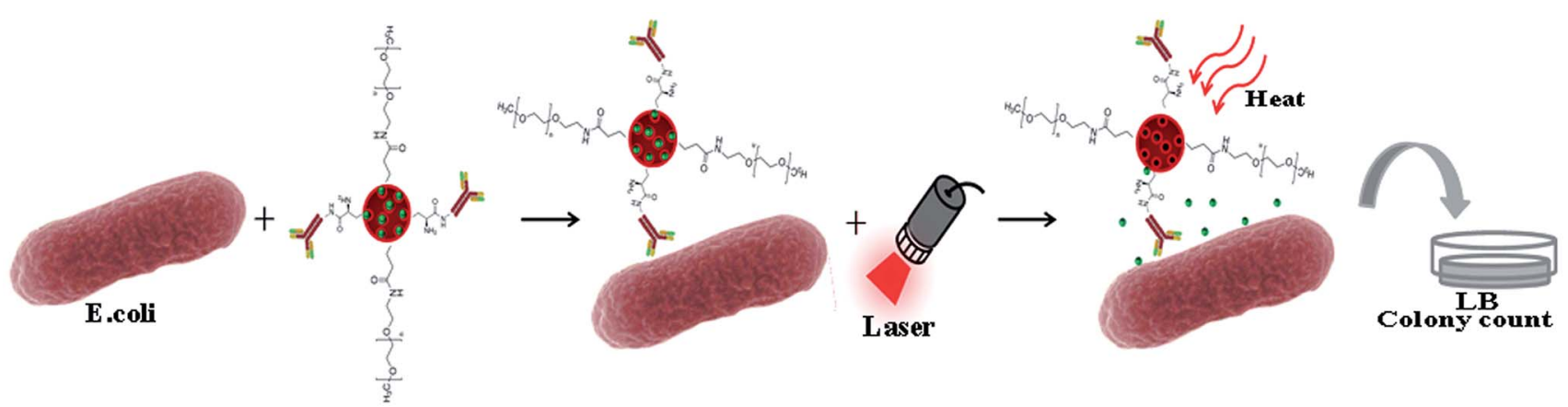

Scheme 2 The hAuNPs-anti-E. coli-kana complexes effectively lead to specific bacterial death through the synergetic effects of kanamycin release and heat generation. 
Table 1 The survival rate of $E$. coli in the presence of various conditions (irradiation at $650 \mathrm{~nm}$ laser, $40 \mathrm{~min}$ )

\begin{tabular}{|c|c|c|c|c|}
\hline & Bacteria (blank) & Hollow Au (laser) & Kanamycin & Hollow Au-kana (laser) \\
\hline Culture medium & LB & LB & LB & LB \\
\hline Culture temperature & $37^{\circ} \mathrm{C}$ & $37^{\circ} \mathrm{C}$ & $37^{\circ} \mathrm{C}$ & $37^{\circ} \mathrm{C}$ \\
\hline Culture time & $20 \mathrm{~h}$ & $20 \mathrm{~h}$ & $20 \mathrm{~h}$ & $20 \mathrm{~h}$ \\
\hline Bacteria viability (\%) & $100.00 \pm 2.90$ & $90.18 \pm 2.80$ & $87.27 \pm 1.83$ & $50.36 \pm 1.76$ \\
\hline Colony images & & & & \\
\hline
\end{tabular}

of colonies. Next, we did a control test under the same experimental conditions, measuring the viability of bacteria (blank), bacteria exposed to physical sterilization with hAuNPs under laser irradiation, the chemical drug sterilization by kanamycin, and the viability of bacteria with the hollow hybrid loaded with kanamycin under laser irradiation. The survival rate of the bacteria after treatment and the images of $E$. coli colony growth on the solid medium are shown in Table 1, with the corresponding histogram shown in Fig. 6. The viabilities of bare $E$. coli, those after exposure to hAuNPs under irradiation, after incubation with bare kanamycin, or after incubation with the hollow hybrid carrying kanamycin, were $100.00 \pm 2.90 \%, 90.18$ $\pm 2.80 \%, 87.27 \pm 1.83 \%$, and $50.36 \pm 1.76 \%$, respectively. The bacterial viability was significantly decreased to $50.36 \pm 1.76 \%$ after incubating with the hAuNPs-anti-E. coli-kana and exposure to irradiation ( $650 \mathrm{~nm}, 200 \mathrm{~mW} \mathrm{~cm}{ }^{-2}$, diameter $2 \mathrm{~mm}, 40 \mathrm{~min}$ ). This result showed that a sterilizing rate of $49.64 \%$ was achieved for the hAuNPs-anti-E. coli-kana under irradiation. This clearly shows that there was a synergistic effect between hAuNPs-anti$E$. coli-kana for each component of the bactericidal effect, producing greatly improved bactericidal properties against $E$.

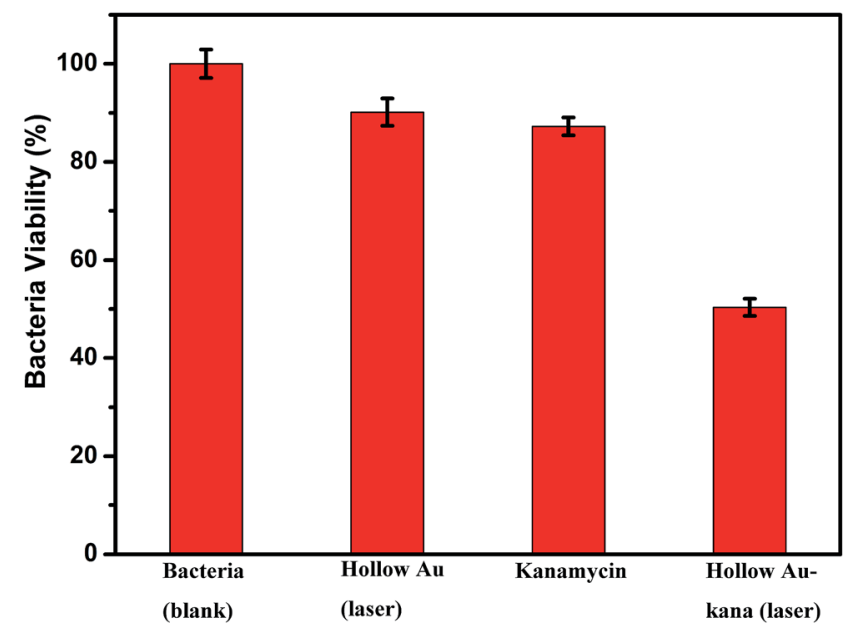

Fig. 6 The corresponding histogram of the survival rate of $E$. coli under various conditions.

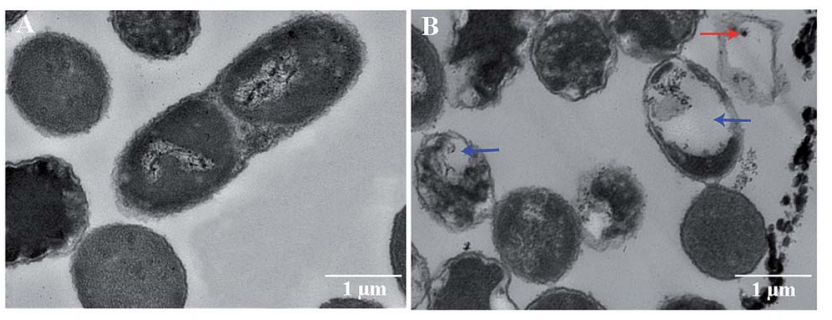

Fig. 7 Morphology of E. coli without and with hAuNPs-anti-E. colikana treatment. TEM images of $E$. coli without (A), and with (B).

coli. In the case of the same bactericidal effect, owing to this synergistic effect, the amount of kanamycin was significantly reduced, which could reduce the possibility of drug resistance.

Here, using $E$. coli as a model, hAuNPs-anti-E. coli-kana with laser caused damage to the $E$. coli structures. After incubating $E$. coli with hAuNPs-anti-E. coli-kana for $4 \mathrm{~h}$, the loss of interior structures and the formation of a large-scale, light-colored area were observed (Fig. 7B). The TEM images indicate that hAuNPsanti-E. coli-kana could be internalized and located inside the cell wall of bacteria (red arrow in Fig. 7B), which seriously destroyed the cell wall and resulted in significant leakage of the cell contents (blue arrows in Fig. 7B).

\section{Conclusion}

In this study, anti-E. coli-conjugated and kanamycin-loaded hAuNPs (hAuNPs-anti-E. coli-kana) were prepared for bacterial targeting and drug delivery. The system has multiple characteristics: dual-ligand modified hAuNPs targeting specific bacteria via the anti-E. coli, and synergistic bactericidal effects of the chemical drug and physical sterilization. In particular, the hollow interior of these NPs allows a significant increase in kanamycin loading. Compared with drug and photothermal treatment alone, the bactericidal rate of the combined treatment with the hAuNPs-anti-E. coli-kana was much higher. Thus, hAuNP-derived nanocarriers are a promising drug-targeting delivery system. 


\section{Acknowledgements}

This work was financially supported by the National Natural Science Foundation of China (81171415) and the Chongqing Municipal Education Commission Science and Technology Research Project Foundation of China (KJ1400211).

\section{Notes and references}

1 J. Davies, EMBO Rep., 2008, 9, S18-S21.

2 Y. Feng, W. Chen, Y. Jia, Y. Tian, Y. Zhao, F. Long, Y. Rui and X. Jiang, Nanoscale, 2016, 8, 13223-13227.

3 P. Chairatana, T. Zheng and E. M. Nolan, Chem. Sci., 2015, 6, 4458-4471.

4 H. K. Pedersen, V. Gudmundsdottir, H. B. Nielsen, T. Hyotylainen, T. Nielsen, B. A. Jensen, K. Forslund, F. Hildebrand, E. Prifti, G. Falony, E. Le Chatelier, F. Levenez, J. Dore, I. Mattila, D. R. Plichta, P. Poho, L. I. Hellgren, M. Arumugam, S. Sunagawa, S. Vieira-Silva, T. Jorgensen, J. B. Holm, K. Trost, H. I. T. C. Meta, K. Kristiansen, S. Brix, J. Raes, J. Wang, T. Hansen, P. Bork, S. Brunak, M. Oresic, S. D. Ehrlich and O. Pedersen, Nature, 2016, 535, 376-381.

5 K. Lewis and P. Strandwitz, Nature, 2016, 535, 501-502.

6 B. Hu, L. P. Zhang, X. W. Chen and J. H. Wang, Nanoscale, 2013, 5, 246-252.

7 A. M. Schwartzberg, T. Y. Olson, C. E. Talley and J. Z. Zhang, J. Phys. Chem. B, 2006, 110, 19935-19944.

8 J. You, R. Zhang, G. Zhang, M. Zhong, Y. Liu, C. S. Van Pelt, D. Liang, W. Wei, A. K. Sood and C. Li, J. Controlled Release, 2012, 158, 319-328.

9 W. Lu, Q. Huang, G. Ku, X. Wen, M. Zhou, D. Guzatov, P. Brecht, R. Su, A. Oraevsky, L. V. Wang and C. Li, Biomaterials, 2010, 31, 2617-2626.

10 Z. V. Feng, I. L. Gunsolus, T. A. Qiu, K. R. Hurley, L. H. Nyberg, H. Frew, K. P. Johnson, A. M. Vartanian, L. M. Jacob, S. E. Lohse, M. D. Torelli, R. J. Hamers, C. J. Murphy and C. L. Haynes, Chem. Sci., 2015, 6, 51865196.

11 I. H. El-Sayed, X. Huang and M. A. El-Sayed, Nano Lett., 2005, 5, 829-834.

12 I. H. Li, H. J. Zhou, J. X. Wang, D. H. Wang, R. X. Shen, X. L. Zhang, P. Jin and X. Y. Liu, Nanoscale, 2016, 8, 11907-11923.

13 I. Yang, H. Shen, X. Zhang, Y. Tao, H. Xiang and G. Xie, Biosens. Bioelectron., 2016, 77, 1119-1125.

14 I. C. Wu, A. R. Deokar, J. H. Liao, P. Y. Shih and Y. C. Ling, ACS Nano, 2013, 7, 1281-1290.

15 I. Dai, X. Cao, K. F. Liu, C. X. Li, G. F. Zhang, L. H. Deng, C. L. Si, J. He and J. D. Lei, J. Mater. Chem. B, 2015, 3, 3754-3766.

16 D. H. Tsai, S. Elzey, F. W. Delrio, A. M. Keene, K. M. Tyner, J. D. Clogston, R. I. Maccuspie, S. Guha, M. R. Zachariah and V. A. Hackley, Nanoscale, 2012, 4, 3208-3217.

17 T. Huang, P. D. Nallathamby, D. Gillet and X. H. Xu, Anal. Chem., 2007, 79, 7708-7718.
18 J. Nicolas, S. Mura, D. Brambilla, N. Mackiewicz and P. Couvreur, Chem. Soc. Rev., 2013, 42, 1147-1235.

19 E. Tegou, M. Magana, A. E. Katsogridaki, A. Ioannidis, V. Raptis, S. Jordan, S. Chatzipanagiotou, S. Chatzandroulis, C. Ornelas and G. P. Tegos, Biomaterials, 2016, 89, 38-55.

20 N. S. Noh, S. Lee, H. Kang, J. K. Yang, H. Lee, D. Hwang, J. W. Lee, S. Jeong, Y. Jang, B. H. Jun, D. H. Jeong, S. K. Kim, Y. S. Lee and M. H. Cho, Biomaterials, 2015, 45, 81-92.

21 A. K. Singh, D. Senapati, S. Wang, J. Griffin, A. Neely, P. Candice, K. M. Naylor, B. Varisli, J. R. Kalluri and P. C. Ray, ACS Nano, 2009, 3, 1906-1912.

22 J. L. Tang, X. M. Jiang, L. M. Wang, H. Zhang, Z. J. Hu, Y. Liu, X. C. Wu and C. Y. Chen, Nanoscale, 2014, 6, 3670-3678.

23 Z. B. Li, H. Huang, S. Y. Tang, Y. Li, X. F. Yu, H. Y. Wang, P. H. Li, Z. B. Sun, H. Zhang, C. L. Liu and P. K. Chu, Biomaterials, 2016, 74, 144-154.

24 B. K. Wang, X. F. Yu, J. H. Wang, Z. B. Li, P. H. Li, H. Wang, L. Song, P. K. Chu and C. Li, Biomaterials, 2016, 78, 27-39.

25 R. R. Xing, K. Liu, T. F. Jiao, N. Zhang, K. Ma, R. Y. Zhang, Q. L. Zou, G. H. Ma and X. H. Yan, Adv. Mater., 2016, 28, 3669-3676.

26 J. B. Qin, Z. Y. Peng, B. Li, K. C. Ye, Y. X. Zhang, F. K. Yuan, X. R. Yang, L. J. Huang, J. Q. Hu and X. W. Lu, Nanoscale, 2015, 7, 13991-14001.

27 X. Y. Zhang, C. Y. Yang, J. P. Zhou and M. R. Huo, Small, 2016, 12, 3578-3590.

28 L. M. Wang, Q. Sun, X. Wang, T. Wen, J. J. Yin, P. Y. Wang, R. Bai, X. Q. Zhang, L. H. Zhang, A. H. Lu and C. Y. Chen, J. Am. Chem. Soc., 2015, 137, 1947-1955.

29 H. Q. Chen, B. Wang, D. Gao, M. Guan, L. N. Zheng, H. Ouyang, Z. F. Chai, Y. L. Zhao and W. Y. Feng, Small, 2013, 9, 2735-2746.

30 Y. Y. Zhao and X. Y. Jiang, Nanoscale, 2013, 5, 8340-8350.

31 K. L. Knappenberger-J, A. M. Schwartzberg, A. M. Dowgiallo and C. A. Lowman, J. Am. Chem. Soc., 2009, 131, 1389213893.

32 C. Loo, A. Lowery, N. Halas, J. West and R. Drezek, Nano Lett., 2005, 5, 709-711.

33 K. J. Prashant, S. L. Kyeong, H. E. S. Ivan and A. E. S. Mostafa, J. Phys. Chem. B, 2006, 110, 7238-7248.

34 L. M. Pan, J. N. Liu, Q. J. He and J. L. Shi, Adv. Mater., 2014, 26, 6742-6748.

35 Z. Z. Wang, Z. W. Chen, N. Gao, J. S. Ren and X. G. Qu, Small, 2015, 11, 4970-4975.

36 C. Chelsea, W. T. Alexander and C. B. Guillermo, Chem. Sci., 2016, 7, 2023-2029.

37 F. Wang, Y. C. Wang, S. Dou, M. H. Xiong, T. M. Sun and J. Wang, ACS Nano, 2011, 5, 3679-3692.

38 H. Hillaireau and P. Couvreur, Cell. Mol. Life Sci., 2009, 66, 2873-2896.

39 G. Sahay, D. Y. Alakhova and A. V. Kabanov, J. Controlled Release, 2010, 145, 182-195.

40 X. K. Zhang, L. J. Meng, Q. H. Lu, Z. F. Fei and P. J. Dyson, Biomaterials, 2009, 30, 6041-6047. 